\title{
Structure and magnetic properties for FePt thin films prepared on $\mathrm{MgAl}_{2} \mathrm{O}_{4}$ and $\mathrm{MgO}$ substrates
}

\author{
H. Iwama, M. Doi and T. Shima \\ Graduate School of Engineering, Tohoku Gakuin University, Tagajo, 985-8537, Japan
}

\begin{abstract}
In order to investigate the effect of lattice mismatch between FePt thin films and single crystal substrates on the tetragonality and the magnetization process, FePt thin films have been fabricated on $\mathrm{MgAl}_{2} \mathrm{O}_{4}(\mathrm{MAO})$ (100) and $\mathrm{MgO}$ (100) single crystalline substrates at a substrate temperature of $700{ }^{\circ} \mathrm{C}$. The Fe concentration in the FePt films was varied from 45.0 to 50.8 at. \%. In addition to the fundamental (002) peak, (001) and (003) superlattice peaks have clearly been observed in the X-ray diffraction patterns for all the samples, indicating the formation of $L 1_{0}$ ordered structure. The magnetization measurements show that all the samples are perpendicularly magnetized. Large coercivity $\left(H_{\mathrm{c}}\right)$ of $57.8 \mathrm{kOe}$ and $54.4 \mathrm{kOe}$ was observed for the films with $\mathrm{Fe}_{49.3} \mathrm{Pt}_{50.7}$ and $\mathrm{Fe}_{48.3} \mathrm{Pt}_{51.7}$ (at. \%) deposited on $\mathrm{MgO}$ and $\mathrm{MAO}$ substrates, respectively. It was confirmed that good hard magnetic properties can be obtained for the $\mathrm{Fe}_{49} \mathrm{Pt}_{51}$ (at. \%) thin film.
\end{abstract}

Key words: $L 1_{0}$ ordered $\mathrm{FePt}$, hard magnetic material, thin film, $\mathrm{MgO}$ substrate, $\mathrm{MgAl}_{2} \mathrm{O}_{4}$ substrate

\section{Introduction}

The magnetization process of assemblies of ferromagnetic nanoparticles with a large uniaxial magnetocrystalline anisotropy is of great scientific and technological interest, since they are expected to be applied in forthcoming magnetic devices such as high density magnetic recording media and high performance biasing nano-magnets. It is well known that the magnetization process and the coercivity depend strongly on the characteristic size and the morphology of the assemblies of ferromagnetic particles. Since FePt alloy shows high uniaxial magnetocrystalline anisotropy $\left(K_{\mathrm{u}}=7.0 \times 10^{7} \mathrm{erg} / \mathrm{cm}^{3}\right) \quad[1]$, moderate saturation magnetization and high corrosion resistance, several studies have recently been done on $L 1_{0}$ ordered $[\mathrm{CuAu}$ (I) type] FePt thin films [2-14] and nanoparticles [15, 16] using conventional thin film preparation methods and chemical syntheses. It is also thought to overcome the instability of magnetization vectors caused by thermal fluctuation even in the nano-meter scaled particles. However, magnetization process of FePt thin films has not been fully elucidated. The lattice mismatch plays a crucial role in the growth of epitaxial thin films. Generally, $\mathrm{MgO}$ single crystalline substrate has been commonly used to induce the $L 1_{0}$ ordered (001) texture of FePt films. Recently, low temperature fabrication of epitaxial $\mathrm{FePt}$ thin films below $400{ }^{\circ} \mathrm{C}$ have been reported for $\mathrm{MgAl}_{2} \mathrm{O}_{4}$ [17] single crystalline substrates. However, systematic investigations of the effects of lattice mismatch on the structure and magnetic properties of FePt films have rarely been reported. Therefore, it would be worthwhile to study the structure and magnetic properties of $\mathrm{FePt}$ films prepared on different substrates. For this purpose, two single crystal substrates $\mathrm{MgO}$ ( $\mathrm{NaCl}$-type) and $\mathrm{MgAl}_{2} \mathrm{O}_{4}$ (Spinel-type) were chosen. The values of unstrained lattice mismatch between a-axis of $L 1_{0} \mathrm{FePt}$ ordered alloy and these substrates are $8.4 \%$ for $\mathrm{MgO}$ [18] and $3.8 \%$ for $\mathrm{MgAl}_{2} \mathrm{O}_{4}$ [19].

In this study, in order to investigate the effect of lattice mismatch between FePt thin films and single crystal substrates on the tetragonality and the magnetization process, FePt thin films have been fabricated on $\mathrm{MgAl}_{2} \mathrm{O}_{4}$ (MAO) (100) and $\mathrm{MgO}$ (100) single crystalline substrates, their structure and magnetic properties have been investigated.

\section{Experimental procedure}

All the samples were prepared using an ultrahigh vacuum magnetron sputtering system (ULVAC, QAM4) with co-deposition of $\mathrm{Fe}$ and $\mathrm{Pt}$ directly onto polished single crystalline MgO (100) or MAO (100) substrates. The lattice parameters of $L 1_{0}$ ordered FePt alloy, MAO (100) substrate and $\mathrm{MgO}$ (100) substrate are aFePt $=$ $0.385 \mathrm{~nm}, c_{\mathrm{FePt}}=0.371 \mathrm{~nm}\left(L 1_{0}-\mathrm{Fe}_{50} \mathrm{Pt}_{50}(\right.$ at. \%) $)$ [20], $a_{\mathrm{MAO}}=0.404 \mathrm{~nm}[19]$ and $a_{\mathrm{MgO}}=0.420 \mathrm{~nm}$ [18]. The targets were commercial products with purities higher than 99.99 at\% for $\mathrm{Fe}$ and 99.9 at\% for Pt. The base pressure was under $8.5 \times 10^{-7} \mathrm{~Pa}$. High-purity argon of $0.2 \mathrm{~Pa}$ was introduced during sputtering. The substrates were heated to $T_{\mathrm{s}}=700{ }^{\circ} \mathrm{C}$ during deposition. The nominal thickness of FePt layer was fixed at $10 \mathrm{~nm}$. The compositions of the films were determined by an electron probe X-ray microanalysis (EPMA) and $X=$ 45.0, 47.2, 48.3, 48.8, 49.3, 49.850 .3 and 50.8 for $\mathrm{Fe}_{X} \mathrm{Pt}_{100-X}$ (at. \%) are confirmed. The structural analysis was performed by X-ray diffraction (XRD) with $\mathrm{Cu} \mathrm{Ka}$ radiation. The lattice constant of $c$-axis was obtained by the out-of-plane XRD measurement, while the value of a-axis was obtained by the in-plane XRD measurement. The film morphology was observed by atomic force microscopy (AFM, in tapping mode). The magnetic properties were measured by a superconducting quantum interference device (SQUID) magnetometer in the field up to $70 \mathrm{kOe}$ at room temperature (R. T.). 


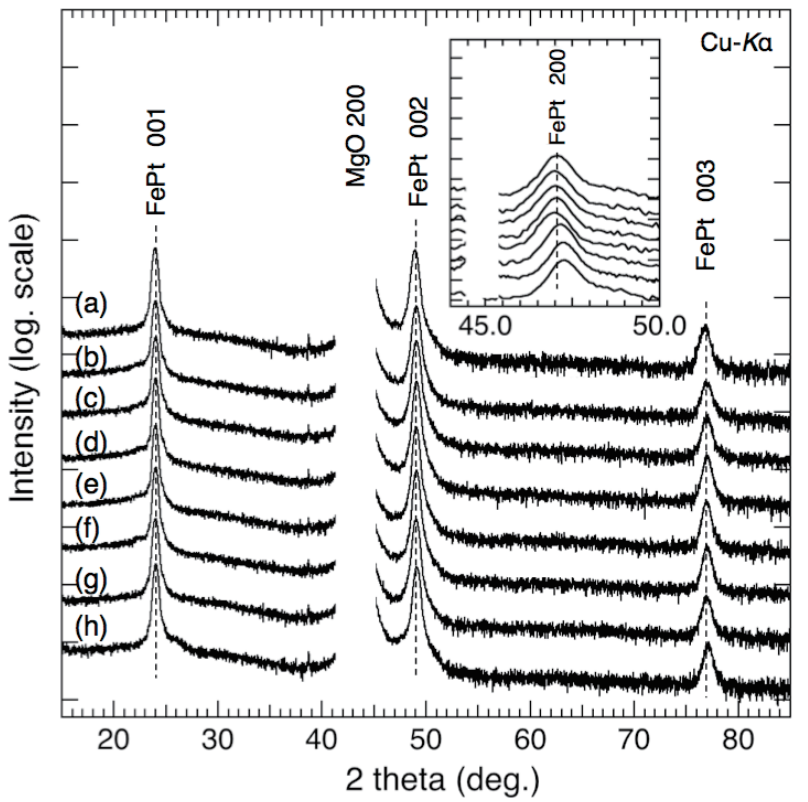

Fig. 1. XRD patterns for FePt thin films prepared on $\mathrm{MgO}$ (100) substrate. Inset show the in-plane XRD patterns for FePt thin films. The Fe content $X$ for $\mathrm{Fe}_{X} \mathrm{Pt}_{100-X}$ films are 45.0 (a), 47.2 (b), 48.3 (c), 48.8 (d), 49.3 (e), 49.8 (f), 50.3 (g) and 50.8 (at. \%) (h)

\section{Results and discussion}

XRD patterns for $\mathrm{Fe}_{X} \mathrm{Pt}_{100-X}$ films with various $\mathrm{Fe}$ content prepared on $\mathrm{MgO}$ (100) substrate are shown in Fig. 1. The Fe content was varied as follows: $X=45.0$ (a), 47.2 (b), 48.3 (c), 48.8 (d), 49.3 (e), 49.8 (f), 50.3 (g) and 50.8 (at. \%) (h). In addition to a fundamental (002) peak, (001) and (003) superlattice peaks of the $L 1_{0}$-FePt phase were clearly observed for all the samples. The peak from the other plane of the $L 1_{0}$ structure was not observed. Therefore, it is confirmed that the $c$-axis of FePt layers was aligned perpendicular to the film plane for the FePt films. With increasing $X$, the peak position of the (001) plane shifts to a higher angle, suggesting that the lattice parameter of $c$-axis decreases due to the change of the tetragonality of $L 1_{0}$ ordered FePt phase. From the integrated intensities of fundamental and superlattice peaks extracted from numerical fitting, the degree of long-range chemical order parameter $S$ was evaluated. The detailed procedure for the evaluation of $S$ was described in a previous letter [21]. The $S$ shows the maximum of $0.80 \pm 0.05$ for $\mathrm{Fe}_{49.3} \mathrm{Pt}_{50.7}$ (at. \%) (e).

Magnetization curves for the $\mathrm{Fe}_{X} \mathrm{Pt}_{100-X}$ films prepared on $\mathrm{MgO}$ (100) substrate are shown in Fig. 2. All the measurements were preformed at R.T.. The solid and broken lines denote the magnetic fields applied in the perpendicular direction to the film plane and in-plane directions, respectively. The magnetic easy axes are perpendicular to the film plane for all the samples, since the [001] axis of the tetragonal $L 1_{0}$ ordered structure was perpendicular to the film plane as

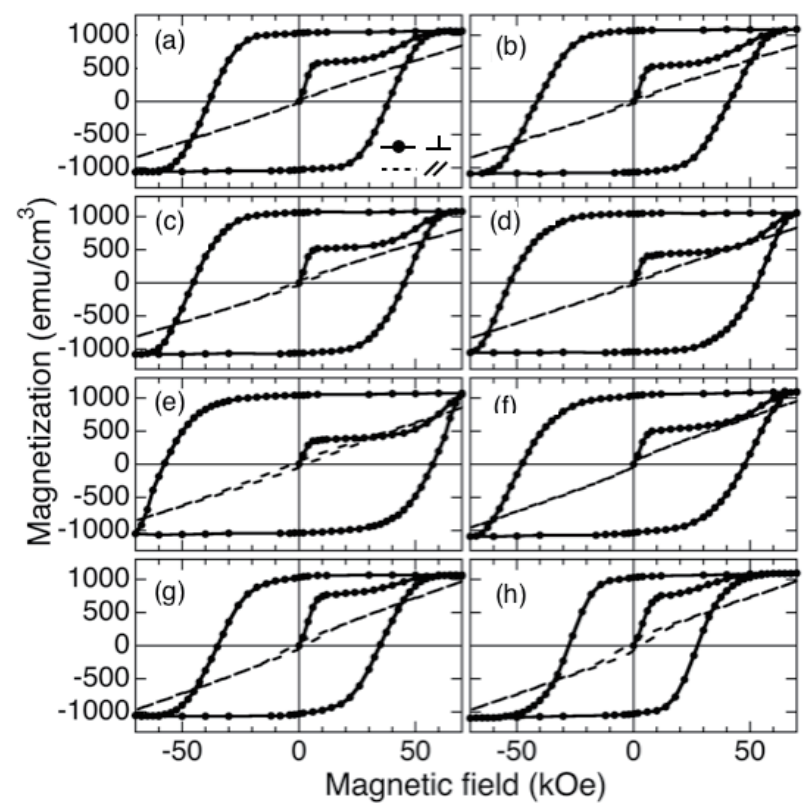

Fig. 2. Magnetization curves for FePt thin films prepared on $\mathrm{MgO}$ (100) substrate. The Fe content $X$ for $\mathrm{Fe}_{X} \mathrm{Pt}_{100-} X$ films are 45.0 (a), 47.2 (b), 48.3 (c), 48.8 (d), 49.3 (e), 49.8 (f), $50.3(\mathrm{~g})$ and 50.8 (at. \%) (h).

demonstrated in Fig. 1. Coercivity $\left(H_{\mathrm{c}}\right)$ of $38.4 \mathrm{kOe}$ was obtained for the film with $X=45.0$ (at. \%) (a). The maximum $H_{c}$ of $57.8 \mathrm{kOe}$ was obtained for the film with $X=49.3$ (at. \%) (e). With further increasing of $\mathrm{Fe}$ content, $H_{c}$ decreases slowly, but still keeps a quite large value of $48 \mathrm{kOe}$ for the film with $X=49.8$ (at. \%) (f). However, $H_{\mathrm{c}}$ decreased to $27.7 \mathrm{kOe}$ for the film with $X=50.8$ (at. \%) (h). The in-plane magnetization does not saturate even at a magnetic field of $70 \mathrm{kOe}$, which is the maximum magnetic field of the SQUID magnetometer, indicating that they possess high uniaxial magnetic anisotropy. A remarkable step in the initial magnetization curve was observed for all samples. A steep increase of the magnetization at low magnetic field was observed. The fractional magnetization at low magnetic field corresponds to the magnetic domain wall displacement; in other words, particles larger than 200 $\mathrm{nm}$ (single domain size [22]) with multiple domain structure are magnetized at low magnetic field. However, it is to be magnetized at applied magnetic field more then $10 \mathrm{kOe}$, because they contain single domain particles. Hence, the magnetization process governed by the magnetic domain wall displacement in the multiple domain particles and the rotation of the magnetization in the single domain particles.

XRD patterns for $\mathrm{Fe}_{X} \mathrm{Pt}_{100-X}$ films prepared on MAO (100) substrate are shown in Fig. 3. The Fe content was varied as follows: $X=45.0(\mathrm{a}), 47.2(\mathrm{~b}), 48.3(\mathrm{c}), 48.8$ (d), 49.3 (e), 49.8 (f), 50.3 (g) and 50.8 (at. \%) (h). Both fundamental and superlattice peaks of the $L 1_{0}-\mathrm{FePt}$ phase were clearly observed for all the samples. The unlabeled peaks were due to the MAO (100) substrate. 


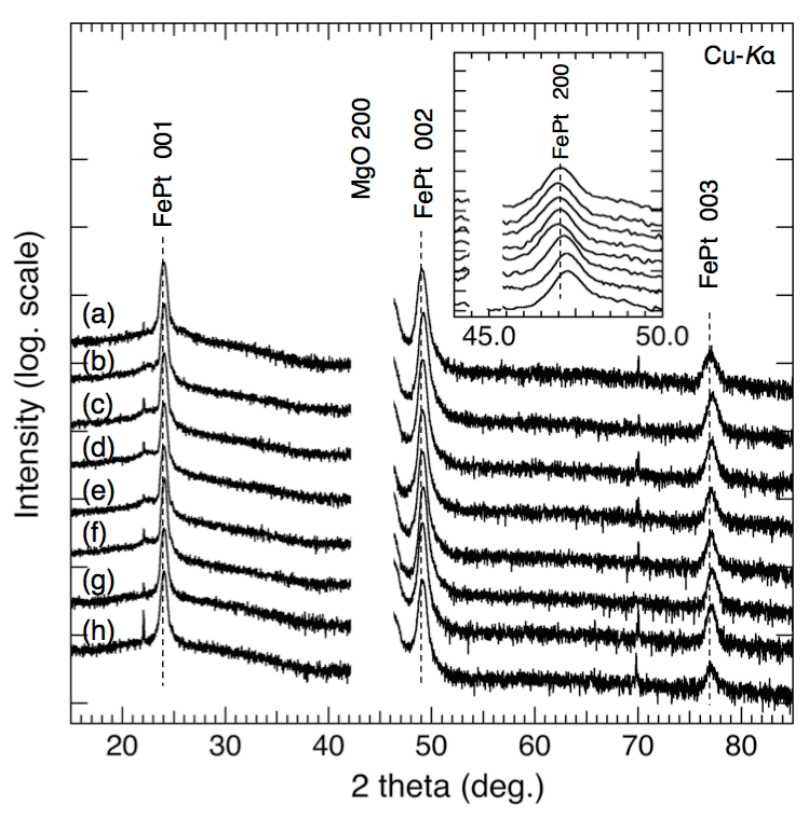

Fig. 3. XRD patterns for FePt thin films prepared on $\mathrm{MgAl}_{2} \mathrm{O}_{4}$ (100) substrate. Inset show the in-plane XRD patterns for FePt thin films. The Fe content $X$ for $\mathrm{Fe}_{X} \mathrm{Pt}_{100-X}$ films are 45.0 (a), 47.2 (b), 48.3 (c), 48.8 (d), 49.3 (e), 49.8 (f), 50.3 (g) and 50.8 (at. \%) (h).

The peak from the other plane of the $L 1_{0}$ structure was not observed. It is also confirmed that $c$-axis of $\mathrm{FePt}$ layers was successfully aligned perpendicular to the film plane for the films deposited on the MAO (100) substrate. This indicates that the highly (001) oriented $L 10$ structure was achieved for the FePt films, even with different $\mathrm{Fe}$ content of the $\mathrm{FePt}$ layer. From the integrated intensities of fundamental and superlattice peaks extracted from numerical fitting, the degree of long-range chemical order parameter $S$ was evaluated. The $S$ shows the maximum of $0.74 \pm 0.05$ for $\mathrm{Fe}_{48.3} \mathrm{Pt}_{51.7}$ (at. \%) (c). The clear four fold symmetry was also confirmed in both of FePt thin films on $\mathrm{MgO}$ (100) and MAO (100) substrates by $2 \theta X / \varphi$ scan of XRD. As a result, the epitaxial growth of $\mathrm{FePt}$ thin films on $\mathrm{MgO}$ (100) and MAO (100) substrates were confirmed. From the AFM observation, island growth with the island size of about $150 \mathrm{~nm}$ was observed in both the FePt thin films on the MgO (100) and the MAO (100) substrates.

Magnetization curves for $\mathrm{FePt}$ films with various Fe content prepared on MAO (100) substrate are shown in Fig. 4. The magnetic field was applied in the perpendicular (solid line) and in-plane (broken line) directions to the film. The easy magnetization axis is aligned perpendicular to the film plane for all the samples. The [001] axis of the tetragonal $L 1_{0}$ ordered structure was perpendicular to the film plane as demonstrated in Fig. 3. $H_{c}$ of $44.1 \mathrm{kOe}$ was obtained for the film with $X=45.0$ (at. \%) (a). The maximum $H_{\mathrm{c}}$ of $54.4 \mathrm{kOe}$ was obtained for the film with $X=48.3$ (at. \%) (c). With further increasing Fe content, $H_{\mathrm{c}}$ decreases slowly, but still keeps a quite large value of $52.1 \mathrm{kOe}$ for

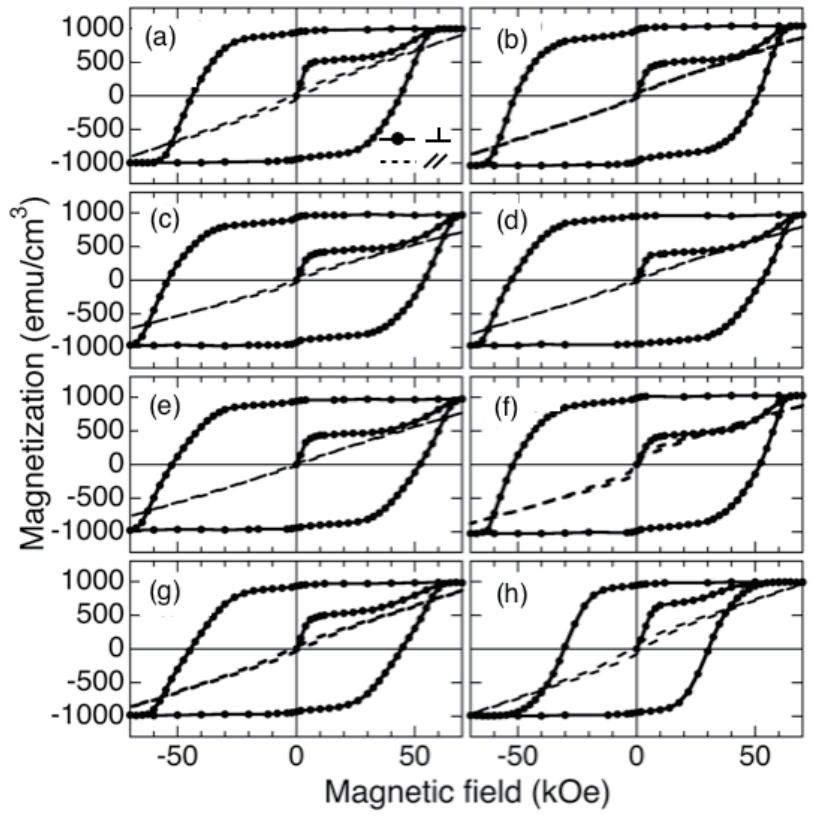

Fig. 4. Magnetization curves for $\mathrm{FePt}$ thin films prepared on $\mathrm{MgAl}_{2} \mathrm{O}_{4}$ (100) substrate. The Fe content $X$ for $\mathrm{Fe}_{X} \mathrm{Pt}_{100-} X$ films are 45.0 (a), 47.2 (b), 48.3 (c), 48.8 (d), 49.3 (e), 49.8 (f), 50.3 (g) and 50.8 (at. \%) (h).

the film with $X=49.8$ (at. \%) (f). However, $H_{\mathrm{c}}$ was decreased to $44.8 \mathrm{kOe}$ and $30.8 \mathrm{kOe}$ for the films with $X$ $=50.3(\mathrm{~g})$ and 50.8 (at. \%) (h), respectively. Similar to the result observed in the initial magnetization curves of the films deposited on $\mathrm{MgO}$ (100) substrate, the magnetization process for the FePt films deposited on MAO (100) substrate is thought to a mixture of a magnetic domain displacement and a rotation of the magnetism.

The a-axis, $c$-axis, $c / a$ and $H_{c}$ for FePt thin films deposited on MgO (100) and MAO (100) substrates with different Fe content are summarized in Fig. 5. Solid circles and solid triangles denote the data for the FePt film deposited on $\mathrm{MgO}$ (100) substrate, while open marks are denote the data for the films deposited on MAO (100) substrate. Maximum value of a-axis was obtained for both substrates at $X=47.1$ (at. \%). However, a-axis was decreased with further increased of Fe content. While monotonical increase was obtained for c/a more than $X=48$ (at. \%) and maximum. $H_{c}$ was obtained at $X=49$ (at. \%) for both films. From these results, it is confirmed that lattice spacing of a-axis is strongly dependent on the composition rather than the one of $c$-axis. The reason why the FePt films deposited on MAO (100) substrates show large $H_{c}$ at wide composition region is thought to arise from a small lattice mismatch between the substrate and the FePt film. The more detail investigation of morphological analysis will be necessary for the exact discussion of $H_{\mathrm{c}}$.

\section{Summary}

The structure and magnetic properties of FePt films 


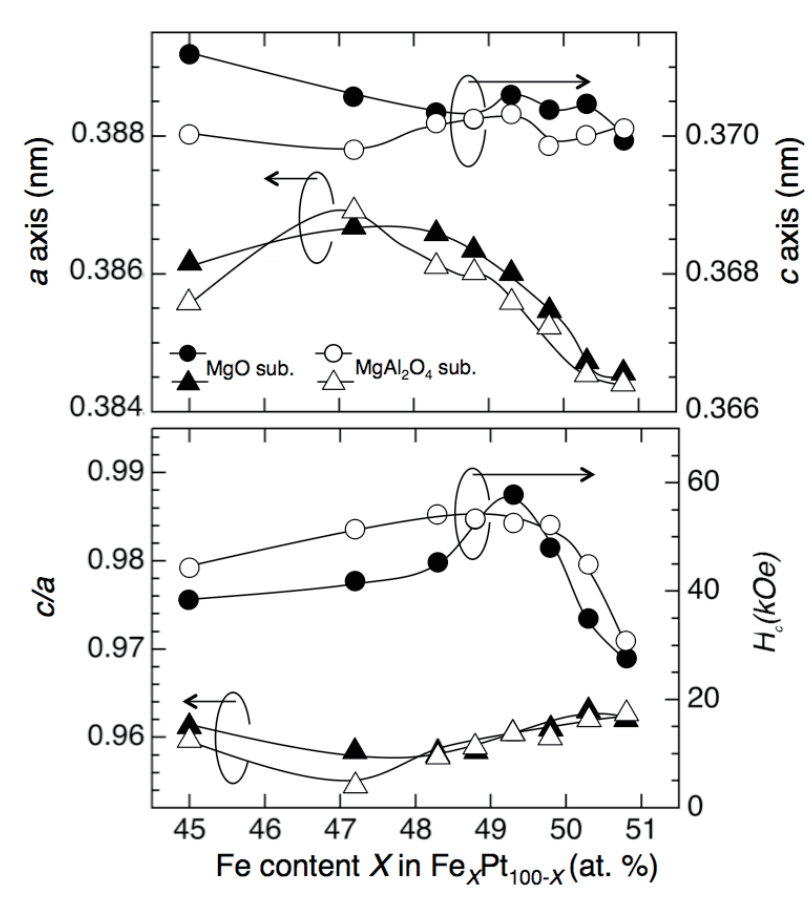

Fig. 5. Effect of Fe content $\mathrm{X}$ on the lattice constant of $a$ and $c$-axis, the tetragonality represented by $c / a$ and coercivity $H_{c}$ for $\mathrm{Fe}_{x} \mathrm{Pt}_{100-X}$ thin films prepared on $\mathrm{MgO}$ (100) and $\mathrm{MgAl}_{2} \mathrm{O}_{4}$ (100) single crystalline substrates.

prepared on $\mathrm{MgO}$ (100) and MAO (100) substrates have been investigated. The FePt layer was epitaxially grown on $\mathrm{MgO}$ (100) and MAO (100) substrates. Large $H_{\mathrm{c}}$ of $57.8 \mathrm{kOe}$ and $54.4 \mathrm{kOe}$ was obtained for the films with $\mathrm{Fe}_{49.3} \mathrm{Pt}_{50.7}$ and $\mathrm{Fe}_{48.3} \mathrm{Pt}_{51.7}$ (at. \%) deposited on $\mathrm{MgO}$ (100) and MAO (100) substrates, respectively. It was confirmed that good hard magnetic properties can be obtained for the $\mathrm{Fe}_{49} \mathrm{Pt}_{51}$ (at. \%) thin film. Although, similar behavior of $H_{c}$ dependence on the composition was observed in FePt thin films on $\mathrm{MgO}$ (100) and MAO (100) substrates, large $H_{c}$ can be obtained at wide composition range of $\mathrm{Fe}_{X} \mathrm{Pt}_{100-X}$ thin films, even at low Fe content between 45.0 and 48.3 (at. \%).

Acknowledgements This work was partially supported by the Research Institute for Engineering and Technology at Tohoku Gakuin University. This work is a cooperative program of the Cooperative Research and Development Center for Advanced Materials, Institute for Materials Research, Tohoku
University.

\section{References}

1) O. A. Ovanov, L. V. Solina, and V. A. Demshina: Phys. Met. Metallogr., 35, 81 (1973).

2) T. Suzuki, K. Harada, N. Honda, and K. Ouchi: Phys. Met. Metallogr., 193, 85 (1999).

3) C. L. Platt, K. W. Wierman, E. B. Svedberg, R. van de Veerdonk, J. K. Howard, A. G. Roy, and D. E. Laughlin: $J$. Appl. Phys., 92, 6104 (2002).

4) T. Shima, K. Takanashi, Y. K. Takahashi, and K. Hono: Appl. Phys. Lett., 81, 1050 (2002).

5) T. Shima, K. Takanashi, Y. K. Takahashi, K. Hono, G. Q. Li, and S. Ishio: Appl. Phys. Lett., 99, 033516 (2006).

6) C. Moutafis, S. Komineas, C. A. F. Vaz, J. A. C. Bland, T. Shima, T. Seki, and K. Takanashi: Phys. Rev. B, 76, 104426 (2007).

7) Z. Xu, X. D. Liu, R. X. Gao, Z. F. Chen, T. S. Lai, H. N. Hu, S. M. Zhou, X. J. Bai, and J. Du: Appl. Phys. Lett., 93, 162509 (2008).

8) T. Narisawa, T. Hasegawa, S. Ishio, and H. Yamane: $J$. Appl. Phys., 109, 033918 (2011).

9) T. Seki, H. Iwama, T. Shima, and K. Takanashi: J. Phys. D: Appl. Phys., 44, 335001 (2011).

10) S. Matsumoto, and T. Shima: J. Phys. Conf. Ser., 266, 012038 (2011).

11) S. Matsumoto, H. Iwama, and T. Shima: J. Magn. Soc. Jpn., 36, 86 (2012)

12) K. F. Dong, H. H. Li, and J. S. Chen: J. Magn. Magn. Mater., 347, 165 (2013).

13) K. F. Dong, H. H. Li, and J. S. Chen: J. Appl. Phys., 113, 233904 (2013).

14) J. Wang, S. Hata, Y. K. Takahashi, H. Sepehri-Amin, B. S. D. Ch. S. Varaprasad, T. Shiroyama, T. Schrefl, and K. Hono: Acta. Mater., 91, 41 (2015).

15) S. Yamamoto, Y. Morimoto, T. Ono, and M. Takano: Appl. Phys. Lett., 87, 032503 (2005).

16) T. Shima, K. Takanashi, Y. K. Takahashi, and K. Hono: Appl. Phys. Lett., 88, 063117 (2006).

17) A. Hotta, T. Ono, M. Hatayama, K. Tsumura, N. Kikuchi, S. Okamoto, and T. Shimatsu: J. Appl. Phys., 115, 17B712 (2014).

18) K. Wasa, M. Kitabatake, and H. Adachi: THIN FILM MATERIALS TECHNOLOGY Sputtering of Compound Materials, (2004) Springer Verlag, Heidelbrg-New York.

19) W. F. Gale, and T. C. Totemeler: Smithells Metals Reference Book (2004) ELSEVIER, Oxford.

20) K. H. J. Buschow: Handbook of Magnetic materials 19, (2011) ELSEVIER, Amsterdam.

21) T. Shima, T. Moriguchi, S. Mitani, and K. Takanashi: Appl. Phys. Lett., 80, 288 (2002).

22) T. Shima, K. Takanashi, G. Q. Li, and S. Ishio: Mater. Trans., 44, 8, 1508 (2003).

Received Oct. 19, 2015; Accepted Mar. 24, 2016 